Proceedings

\title{
Disseminated Computation, Cognitive Domestication of New Ignorant Substrates, and Overcomputationalization ${ }^{+}$
}

\author{
Lorenzo Magnani \\ Department of Philosophy and Computational Philosophy Laboratory, University of Pavia, 27100 Pavia, \\ Italy; lmagnani@unipv.it \\ + Conference Morphological, Natural, Analog and Other Unconventional Forms of Computing for Cognition \\ and Intelligence, Berkeley, CA, USA, 2-6 June 2019.
}

Published: 7 May 2020

\begin{abstract}
Eco-cognitive computationalism considers computation in the context of following some of the main tenets advanced by the recent cognitive science views on embodied, situated and distributed cognition. It is in the framework of this eco-cognitive perspective that we can usefully analyze the recent attention in computer science devoted to the importance of the simplification of cognitive and motor tasks caused in organic entities by the morphological features: ignorant bodies can be domesticated to become useful "mimetic bodies", that is to be able to render an intertwined computation simpler, resorting to that "simplexity" of animal embodied cognition, which represents one of the main qualities of organic agents. Through eco-cognitive computationalism we can clearly acknowledge that the concept of computation changes, depending on historical and contextual causes and we can build an epistemological view that illustrates the "emergence" of new kinds of computations, such as the one regarding morphological computation. This new perspective shows how the computational domestication of ignorant entities can originate new unconventional cognitive embodiments. I also introduce the concept of overcomputationalism, showing that my proposed framework helps us see the related concepts of pancognitivism, paniformationalism and pancomputationalism in a more naturalized and prudent perspective, avoiding the excess of oldfashioned ontological or metaphysical overstatements.
\end{abstract}

Keywords: domestication of ignorant entities; mimetic minds; mimetic bodies; eco-cognitive computationalism; morphology; overcomputationalism

\section{Eco-Cognitive Computationalism}

"Disseminated Computation, Cognitive Domestication of New Ignorant Substrates, and Overcomputationalization" first of all aimed at presenting "eco-cognitive computationalism", which sees computation as active in physical entities suitably transformed so that they become what I call "cognitive mediators", in which data can be encoded and decoded to obtain fruitful results. This perspective is related the study of computation in the context of some of the main tenets advanced by the recent cognitive science views on embodied, situated and distributed cognition, an interdisciplinary perspective that I have recently illustrated in my book The Abductive Structure of Scientific Creativity [1]. I indeed think that the main advantage of speaking of the transformation of "ignorant" entities into cognitive ones through computation derives from its capacity to stress the dynamic character of processes of the attribution of cognitive roles to external entities. This idea is certainly related to the studies in the area of evolutionary theories-especially the ones on cognitive niche construction [2]-and to the recent tradition of studies in the area of cognitive science called 
"distributed cognition". Indeed, the idea of cognition that I am adopting is informed by the "ecological" perspective I fully explained and described in previous studies on the concept of abduction.

\section{Domestication of New Substrates: Morphological Computing}

It is in this eco-cognitive perspective that I analyze the recent attention in computer science devoted to the importance of what I call the cognitive domestication of new substrates, such as in the case of morphological computation: this new area of research shows how the computational domestication of ignorant substrates can originate new unconventional cognitive embodiments, which expand the processes of computationalization already occurring in our societies. A recent area of studies that concerns the so-called morphological computation deals with the problem of enriching classical digital computation, taking advantage of "bodies" which are transformed in entities that carry their own computation in a useful (and unusual) way. It is in this framework of research that Hauser et al. [3] (p. 230) say that the body does not "know" that it is part of a computational device because it simply conforms to the laws of physics and it responds accordingly. Moreover, the body does not over- nor under-compensate, indeed, it is a stable physical system. The apparatus at play simply adds some linear readouts to the body to conclude the computation. The body would behave precisely the same, if there were no readouts at all. The authors further note that "If this output is used, e.g., in a feedback loop as a control signal for the robot, the behavior of the robot of course should be different if we close the loop by adding the readout. However, the body still does not 'know' that it is part of this computational loop. This raises the philosophical question, sometimes referred to as the teleological principle, whether the body is carrying out computation at all" (ibid.). Moreover, the so-called Physical RC (Reservoir Computing) [4] is of practical value if it is able to provide a fruitful mapping of input streams onto output streams taking advantage of the physical body at hand. Finally, the authors introduce the role of ignorance: "Another interesting implication of this property of 'ignorance' of the body (i.e., it behaves the same, whether we add readouts or not) is that we can employ the same body for multiple computations on the same input at the same time. We simply have to add the according number of readouts" (ibid.).

\section{Overcomputationalism and Pancognitivism, Paniformationalism, and Pancomputationalism}

I am also introducing, paying attention to a kind of ethical side of the problem of the domestication of ignorant entities, the concept of overcomputationalism, as intertwined with the traditional concepts of pancognitivism, paniformationalism and pancomputationalism, seeing them in a more naturalized intellectual disposition, appropriate to the aim of bypassing or naturalizing ontological or metaphysical overstatements. What I call overcomputationalization refers to the presence of too many entities and artifacts that carry computational tasks and powers. Overcomputationalization (1) often promotes plenty of possible unresolvable disorganizational consequences, and (2) tends to favor philosophical reflections that depict an oversimplified vision of the world. Moreover, it tends to generate too many cognitive constraints and limitations, which lead to a weakening of the human creative (abductive) cognitive activities, as I have illustrated in the last chapter of my book quoted above [1], and because of the excess of redundant cognitive/informational features attributed to entities (features often exogenous to the original functions of them) it tends to prevent human intellectual freedom to benefit from that cognitive simplification that is characteristic of the absence of informational overloads. This may be in the case of various entities, both natural and artificial, the property of being computationally ignorant has to sometimes be protected.

The reader must not misunderstand me, I believe that we should choose carefully what to defend from computationalization and what is good to computationalize, and so my urgency to "protect ignorant entities" does not have to be intended as a warning about all kinds of computationalization. The computational modeling of the human body and brain can for example foster a better understanding of diseases, degenerative processes, aging and other processes of life, including processes in our brains that generate the human mind. The human brain is the largely unknown organ in the human body and a lot of health problems, especially of ageing populations, are related to brains. Just to make few examples, we do not have cures for Alzheimer's, Parkinson's disease or 
Autism. Therefore, fruitful current and future computational modeling will not only favor the further domestication of ignorant entities by transforming them into "skilled" substrates able to carry computational and thus cognitive performances, but will also fecundate scientific research related to the removal of another kind of ignorance, the classical one regarding the lack of understanding of high-level cognitive capacities in both human and nonhuman animals, simpler organisms and other biological entities such as cells, as also hoped by the admonition contained in the recent review "Time to reinspect the foundations? Questioning if computer science is outgrowing its traditional foundations" [5].

Funding: This research was funded by Funds: Blue Sky Research 2017 (Grant No. BSR1780130) - University of Pavia, Pavia, Italy.

Conflicts of Interest: The author declares no conflict of interest.

\section{References}

1. Magnani, L. The Abductive Structure of Scientific Creativity; Springer: Cham, Switzerland, 2017.

2. Odling-Smee, F.J.; Laland, K.N.; Feldman, M.W. Niche Construction. The Neglected Process in Evolution; Princeton University Press: Princeton, NJ, USA, 2003.

3. Horsman, C.; Stepney, S.; Wagner, R.C.; Kendon, V. When does a physical system compute? Proc. R. Soc. A Math. Phys. and Eng. Sci. 2014, 470, 20140182.

4. Müller, V.C.; Hoffmann, M. What is morphological computation? On how the body contributes to cognition and control. Artif. Life 2017, 23, 1-24.

5. Copeland, J.; Dresner, E.; Proudfoot, D.; Shagrir, O. Time to reinspect the foundations? Questioning if computer science is outgrowing its traditional foundations. Commun. ACM 2016, 59, 34-38.

(C) 2020 by the authors. Licensee MDPI, Basel, Switzerland. This article is an open access article distributed under the terms and conditions of the Creative Commons Attribution (CC BY) license (http://creativecommons.org/licenses/by/4.0/). 